\title{
Sub-Nanosecond Phase Recovery in Quantum-Dot Semiconductor Optical Amplifiers
}

\author{
K. ABEDi* AND H. TALEB \\ Department of Electrical Engineering, Faculty of Electrical and Computer Engineering \\ Shahid Beheshti University, G.C. 18993443332, Tehran, Iran
}

\begin{abstract}
In this paper, optimal design of a pumping scheme for achieving the best gain and phase response in quntum-dot semiconductor optical amplifiers is investigated. For the first time, the dynamic response of the quntum-dot semiconductor optical amplifier is evaluated under three different pumping schemes, known as optical pumping, electrical pumping, and electro-optical pumping. Simulation results show that the shortest gain recovery time in quntum-dot semiconductor optical amplifiers can be achieved under the electrical pumping scheme. However, under the optical pumping and electro-optical pumping schemes, the quntum-dot semiconductor optical amplifier represents a shorter phase recovery compared to the electrical pumping scheme. We found that a sub-nanosecond phase recovery in the quntum-dot semiconductor optical amplifier can be achieved under the OP and electro-optical pumping schemes, which can never be achieved under the electrical pumping, because of the slow carrier dynamics of the carrier reservoir. Also, it was found that the gain recovery process in an optically pumped quntum-dot semiconductor optical amplifier can be significantly accelerated at cryogenic temperatures. This result demonstrates the superiorities of the optical pumping scheme over the electrical pumping and electro-optical pumping schemes at low temperatures, where both the gain and phase recovery times of an optically pumped quantum-dot semiconductor optical amplifier are drastically decreased.
\end{abstract}

DOI: 10.12693/APhysPolA.123.407

PACS: 71.55.Eq, 78.67.-n, 78.67.Hc

\section{Introduction}

Quantum-dot semiconductor optical amplifiers (QD-SOAs) have been intensively investigated during the last decade. Both theoretical and experimental studies have proven the unique capabilities of these devices. Ultrafast gain recovery [1-4], low noise figure of $3.5 \mathrm{~dB}[5]$, high saturated output power [5-7], pattern-effect free XGM-based wavelength conversion up to $160 \mathrm{Gbits} / \mathrm{s}$ [8], and capability of operation at Tbits/s speeds in presence of a control signal [9] are among these capabilities.

Optoelectronic devices like QD-SOAs and QD-lasers need a pumping mechanism for generation of a population inversion in their active region. Currently, most researches in the field of QD-SOAs are concentrated on electrically pumped QD-SOAs (EP-QDSOAs). In recent years, the optical pumping (OP) scheme has been introduced as an alternative pumping scheme for QD-SOAs. Theoretical studies have proven distinctive superiorities of optically pumped QD-SOAs (OP-QDSOAs) over their conventional counterparts, i.e. EP-QDSOAs $[9,10]$. Based on these studies, the gain and phase recovery time in an OP-QDSOA can be much less than that in an EP-QDSOA.

Since there are a few works in the literature on the influence of the pumping mechanism on the dynamic response of QD-SOAs, more studies are required to investigate the influence of pumping scheme and operating conditions on dynamic response of QD-SOAs. The dy-

*corresponding author; e-mail: k_abedi@sbu.ac.ir namic model which is employed to evaluate the response of the QD-SOA is based on the state space theory for SOAs $[11,12]$.

The dynamic response of the device is evaluated under both the OP and EP schemes. Also, we investigate the dynamic response of the device under a combination of the OP and EP schemes (EOP scheme). Then, the operations of these pumping schemes are compared with each other and their advantages and disadvantages are discussed in detail. Also, the effect of the temperature on the dynamic of the device is studied. To our knowledge, this is for the first time that effects of the pumping scheme and also the device temperature on the dynamic response of QD-SOAs are investigated.

This paper is organized as follows. The physical structure and theoretical model of the investigated QD-SOA are introduced in Sect. 2. In Sect. 3, dynamic response of the device under different pumping schemes is investigated. Finally, Sect. 4 gives a summary of our work.

\section{Physical structure of the QD-SOA}

The investigated device is an InAs/GaAs QD-SOA which operates around $1.3 \mu \mathrm{m}$. The active region of the QD-SOA consists of ten stacked QD layers which are sandwiched between two AlGaAs cladding layers. Self-assembled QDs in each layer are covered with a 5nm-thick InGaAs capping layer. In addition, QD layers are separated by 33-nm-thick p-doped GaAs spacer layers [4]. The physical structure of the device is illustrated in Fig. 1. Since QDs are grown self-assembly, they have slightly different properties induced by size fluctuations. 


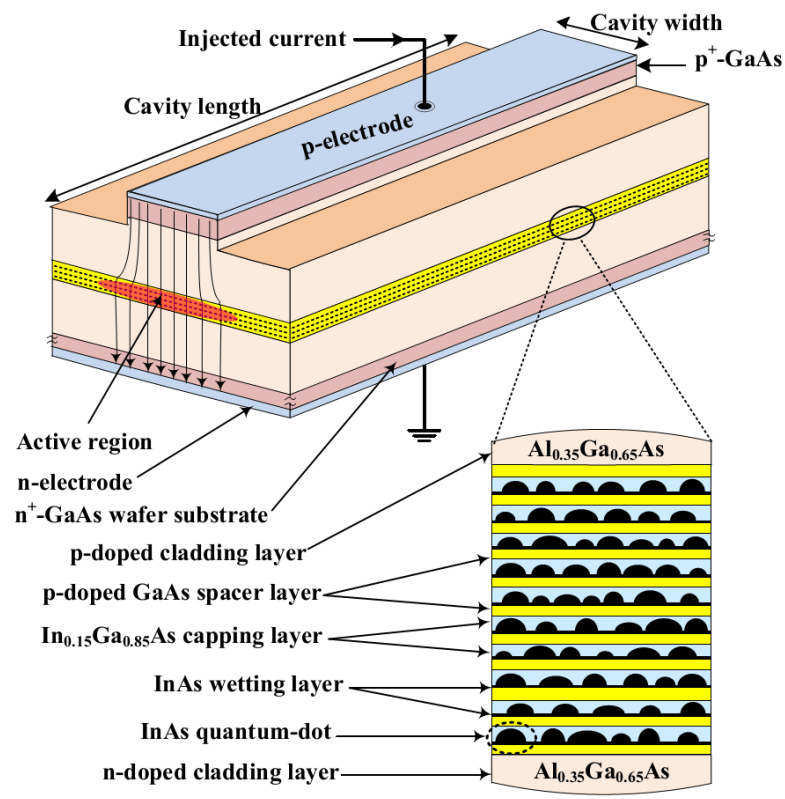

Fig. 1. The physical structure of the investigated QD-SOA.

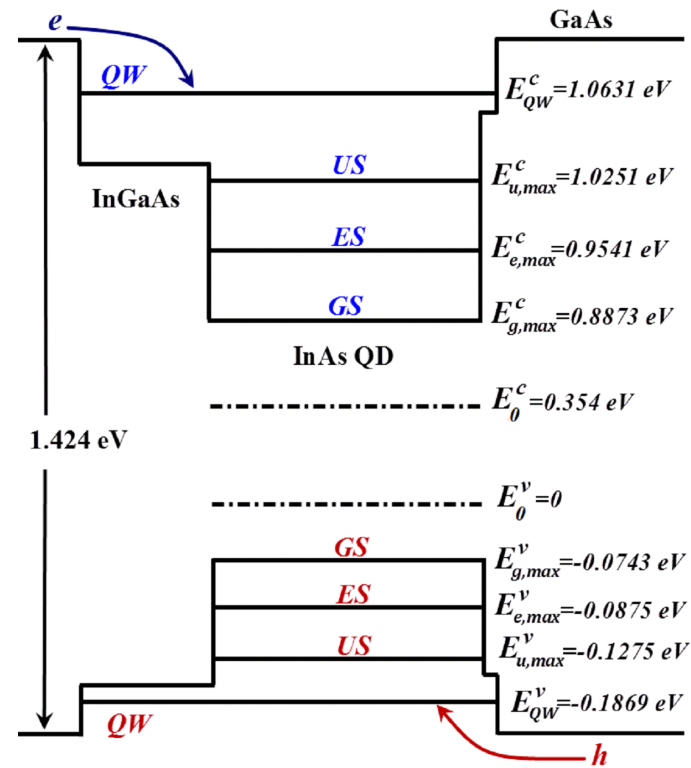

Fig. 2. Energy band diagram of a QD group. Relative energies of the 91-th QD group are designated in the figure.

It is assumed that the self-assembled QDs have a size distribution with a Gaussian profile and consist of 181 spectral groups, where QDs in each QD group are assumed to be identical. The energy band diagram of the $j$-th QD spectral group is shown in Fig. 2, where the relative energy positions of the most probable QD group are designated in the figure [4]. As can be seen in the figure, each QD group has three energy levels in the conduction band
(CB) and three energy levels in the valence band (VB), designated with the ground state (GS), the excited state (ES), and the upper state (US). The effect of the carrier reservoir is modeled by considering a many-fold degenerate energy level in the energy band diagram, which is designated with the QW state.To evaluate the dynamic response of the device, we need a dynamical model for the QD-SOA. For this purpose, we have derived a nonlinear state space model (NSSM) for the device in which the average values of the carrier occupation probabilities are the state variables of the system [12].

\section{Gain and phase response of the QD-SOA under the EP, OP, and EOP schemes}

To investigate the gain and phase recovery time of the QD-SOA under different pumping schemes, we numerically perform a single pulse pump-probe experiment and monitor the dynamic response of the device. It is assumed that the wavelength of the input pulse corresponds to the GS peak, i.e. $\lambda_{\text {pulse }}=1292 \mathrm{~nm}$. A Gaussian shaped pulse is injected into the QD-SOA active region at $t=10 \mathrm{ps}$. The time width and energy of the pulse is assumed to be $0.6 \mathrm{ps}$ and $0.65 \mathrm{pJ}$, respectively.

The optical gain response of the QD-SOA under different pumping schemes is shown in Fig. 3. Under the OP, three different pumping powers are considered: $P_{\text {pump }}=$ 50,100 , and $200 \mathrm{~mW}$. These optical powers are corresponding to the injection current densities of $J=1,2$, and $4 \mathrm{kA} / \mathrm{cm}^{2}$ under the EP [12]. Also, the injection current density under the optimal EOP is $J=346 \mathrm{~A} / \mathrm{cm}^{2}$, which is corresponding to $P_{\text {elec }}=34.6 \mathrm{~mW}$. The remaining power is provided by an optical pump, i.e. the pumping power is $P_{\text {pump }}=15.4,65.4$, and $165.4 \mathrm{~mW}$, respectively. As depicted from the figure, the gain recovery time of the QD-SOA under the EP scheme is shortest compared to the OP and EOP schemes (see Fig. 3b). In addition, while the gain compression is nearly equal in all three schemes, the gain of the QD-SOA is larger under the EP scheme. Consequently, the gain response of the QD-SOA under the EP scheme is better than two other schemes.

One important point about the gain response of the device under the OP scheme is that since the GS transparency power of the QD-SOA is higher than $50 \mathrm{~mW}$, at this power the device cannot be biased at the gain regime. This shortcoming is compensated in the optimal EOP scheme, where the ES transparency is guaranteed by injection of a current with a density of $J=346 \mathrm{~A} / \mathrm{cm}^{2}$. In conclusion, the EP scheme represents the best gain response among three pumping schemes. After that, the EOP scheme has the good gain recovery. Finally, the OP scheme represents the weakest gain response among all pumping schemes. The ultrafast gain recovery of the QD-SOA under the EP is attributed to the spectral hole burning (SHB), which is the dominant gain saturation mechanism at high injection current densities [13]. On the contrary, since the role of the carrier reservoir in the 


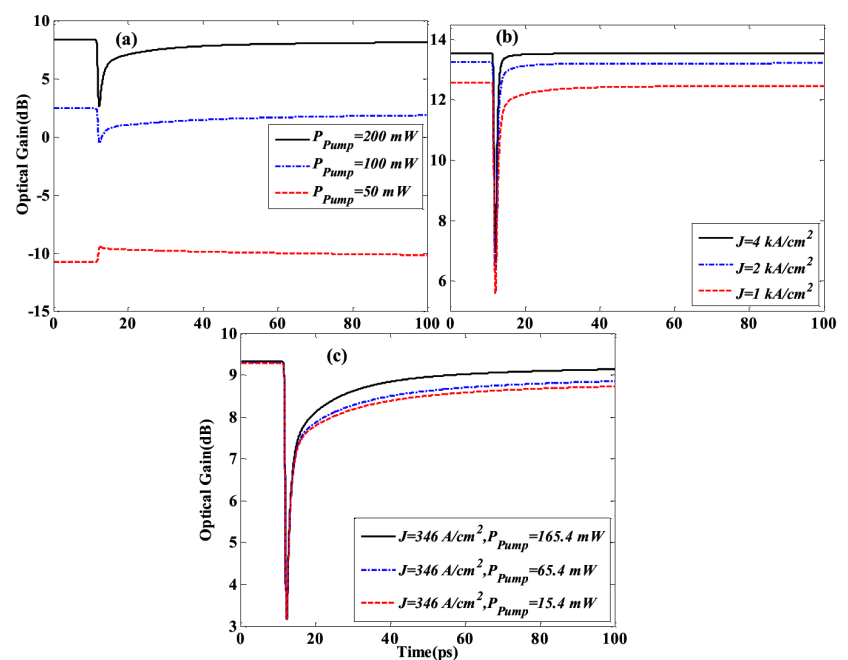

Fig. 3. Optical gain response of the QD-SOA under (a) the OP scheme, (b) the EP scheme, and (c) the optimal EOP scheme.

gain recovery process is diminished under the $\mathrm{OP}$ and EOP schemes, total carrier density depletion (TCDD) is the dominant gain saturation mechanism and a complete gain recovery takes place during a much longer time.

Although the gain responses of SOAs are usually measured in the process, it is insufficient in achieving the optimal SOA performances. In fact, the phase recovery time can be an important factor in operation of SOA-based devices, like all-optical gates.

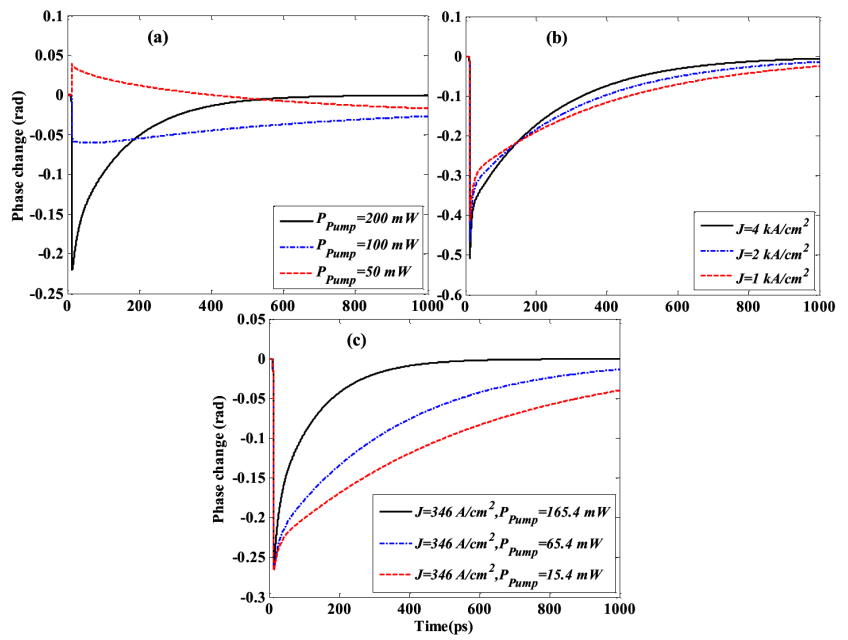

Fig. 4. Phase response of the QD-SOA under (a) the OP scheme, (b) the EP scheme, and (c) the optimal EOP scheme.

In order to compare the phase recovery time of the QD-SOA under different pumping schemes, the phase response of the device is presented in Fig. 4. In contrast to what was observed in the gain response of the device, the EP scheme represents the slowest phase response among all pumping schemes. On the other hand, the phase response of the QD-SOA under the OP and EOP schemes is much better than that under the EP scheme. The slow phase recovery in an electrically pumped QD-SOA is attributed to a significant contribution of the carrier reservoir in the index changes [14]. Since under the OP and EOP schemes the US and the QW state have a negligible effect on the index changes, a superior phase response appears under the OP and EOP schemes.

Up to this point, the gain and phase response of the device was investigated under the OP, the EP, and the EOP schemes. We observed that the EOP scheme represents a superior phase response and a fairly good gain response which make it suitable for applications in all-optical signal processing. However, compared to the EP and OP schemes, implementation of the EOP scheme is more complex. Therefore, we should find a more practical way to overcome this limitation.

Since the phase recovery time in an EP-QDSOA is severely limited by the slow phase recovery induced by the QW carrier reservoir, we should find a method to improve the capabilities of the OP scheme. We observed that the OP scheme cannot be employed to improve the performance of the device at low pumping powers. This was due to the fact that at room temperature both the GS and the ES transparency powers are much higher than the standard pumping powers. In order to reduce the transparency power of an OP-QDSOA, we can reduce the homogeneous broadening (HB) of the device with decrease of the device temperature. With decrease of the $\mathrm{HB}$, the modal gain increases and the transparency power decreases as well. Temperature dependence of the HB is determined by considering the contributions of acoustic phonons, the optical phonons and the zero-temperature lattice motion to the dephasing time [15]. We assume that the device temperature is decreased from the room temperature to a cryogenic temperature $T=77 \mathrm{~K}$. The pumping power is similar to what was considered in the previous simulation, i.e. $P_{\text {pump }}=50,100$, and $200 \mathrm{~mW}$.

The gain and phase responses of the device are presented in Fig. 5a and b, respectively. As can be seen in the figure, both the gain and phase recovery time of the QD-SOA can be significantly decreased at cryogenic temperatures. This interesting result can be explained as follows. At low temperatures the homogeneous broadening is small. For instance, at $T=77 \mathrm{~K}$ the homogeneous linewidth is around $0.31 \mathrm{meV}$ [15]. Consequently, different QD groups have no correlation to each other. Therefore, only a small portion of the self-assembled QDs that their ES transitions are in resonance with the pump power can be activated by the pump signal. In other words, since the pump signal can only be absorbed by one QD group, the carrier capturing process is greatly accelerated and the gain and phase responses of the device are significantly improved. In addition, since the numbers of QD groups participating in the amplification of the input signal decrease with the decrease of the homogeneous linewidth, a pronounced hole burning occurs 
at the energy of input photons and saturation occurs for lower pulse energies.

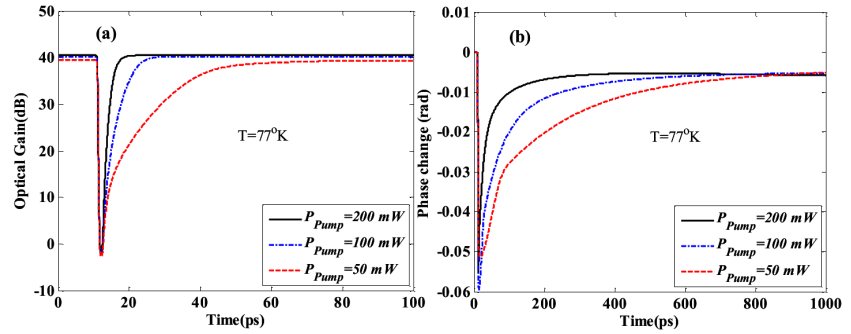

Fig. 5. Dynamic response of the QD-SOA under the $\mathrm{OP}$ at cryogenic temperatures $(T=77 \mathrm{~K})$ : (a) the gain response and $(b)$ the phase response.

This behavior can be observed in Fig. 5a. Although the gain compression is drastically increased, the phase change is significantly diminished. This is due to fact that the number of QD groups which contribute to the amplification of the input pulse decreases for smaller homogeneous linewidths and consequently the changes in the refractive index decrease as well. This result implies that the device temperature is an important factor in optimal operation of an OP-QDSOA, which can be used to control the gain compression and magnitude of phase variations.

\section{Conclusion}

In this paper, for the first time the dynamic response of a QD-SOA under three different pumping schemes (optical, electrical, and electro-optical) was investigated. To evaluate the gain and phase response of the QD-SOA, a NSSM was introduced. Simulation results showed that under equal pumping powers, the gain recovery time of the QD-SOA under the EP scheme is much shorter than that under the OP and EOP schemes. However, the phase recovery of the QD-SOA under the OP and EOP schemes takes place within a shorter time compared to the EP scheme. Also, we found that the gain and phase recovery of the QD-SOA under the OP scheme is accelerated at cryogenic temperatures, which demonstrates the superiorities of the OP scheme over the EP and EOP schemes at low temperatures. The results of this paper are essential for optimal design and fabrication of future all-optical networks.

\section{Acknowledgments}

This work was supported by Shahid Beheshti University under the grant 600-404.

\section{References}

[1] Z. Bakonyi, H. Su, G. Onishchukov, L.F. Lester, A.L. Gray, T.C. Newell, A. Tuennermann, IEEE J. Quantum Electron. 39, 1409 (2003)

[2] H. Taleb, K. Abedi, S. Golmohammadi, Appl. Opt. 50, 608 (2011)

[3] T. Vallaitis, C. Koos, R. Bonk, W. Freude, M. Laemmlin, C. Meuer, D. Bimberg, J. Leuthold, Opt. Exp. 16, 170 (2008)

[4] J. Kim, M. Laemmlin, C. Meuer, D. Bimberg, G. Eisenstein, IEEE J. Quantum Electron. 45, 240 (2009)

[5] J.L. Xiao, Y.Z. Huang, IEEE J. Quantum Electron. 44, 448 (2008)

[6] T.W. Berg, S. Bischoff, I. Magnusdottir, J. Moerk, IEEE Photon. Technol. Lett. 13, 541 (2001)

[7] A.V. Uskov, E.P. O'Reilly, M. Laemmlin, N.N. Ledentsov, D. Bimberg, Opt. Commun. 248, 211 (2005)

[8] G. Contestabile, A. Maruta, S. Sekiguchi, K. Morito, M. Sugawara, K. Kitayama, in: Proc. 35th European Conference on Optical Communication, ECOC 2009, Vienna, Vienna 2009, p. PDP 1,4.

[9] A. Rostami, H.B.A. Nejad, R.M. Qartavol, H.R. Saghai, IEEE J. Quantum Electron. 46, 354 (2010)

[10] H. Baghban, R. Maram, R. Oliaee, R. Yadipour, A. Rostami, J. Opt. 13, 035406 (2011)

[11] S.B. Kuntze, L. Pavel, J.S. Aitchison, IEEE J. Quantum Electron. 43, 123 (2007)

[12] K. Abedi, H. Taleb, J. Lightwave Technol. 30, 1924 (2012)

[13] J. Kim, M. Laemmlin, C. Meuer, D. Bimberg, G. Eisenstein, IEEE J. Quantum Electron. 44, 658 (2008)

[14] J. Kim, C. Meuer, D. Bimberg, G. Eisenstein, Appl. Phys. Lett. 94, 041112 (2009)

[15] G. Ozgur, A. Demir, D.G. Deppe, IEEE J. Quantum Electron. 45, 1265 (2009) 